\title{
Téoros
}

Revue de recherche en tourisme

\section{Destination Méditerranée}

\section{Les rencontres méditerranéennes du tourisme}

\section{Samir Khlifi}

Volume 20, numéro 1, printemps 2001

Mers et littoraux

URI : https://id.erudit.org/iderudit/1071905ar

DOI : https://doi.org/10.7202/1071905ar

Aller au sommaire du numéro

Éditeur(s)

Université du Québec à Montréal

ISSN

0712-8657 (imprimé)

1923-2705 (numérique)

Découvrir la revue

Citer cet article

Khlifi, S. (2001). Destination Méditerranée : les rencontres méditerranéennes du tourisme. Téoros, 20(1), 13-16. https://doi.org/10.7202/1071905ar d'utilisation que vous pouvez consulter en ligne.

https://apropos.erudit.org/fr/usagers/politique-dutilisation/ 


\section{Destination Méditerranée}

\section{Les rencontres méditerranéennes du tourisme}

\section{Samir Khlifi}

Face à la mondialisation, à fa concurrence internationale, des opérateurs se regroupent, des institutions s'associent et des destinations touristiques s'unissent pour former un seal bloc. C'est le cas de destinations comme le Pacifigue et les Caraübes. Aujoud' hui la Méditerranée forme-t-elle une destination à part entiere? Est=ce qu'on voyage davantage en Mediterranee pour visiter I'Espagne ou en Espagne, pays méditerranéen? Le mythe de la Medierranée subsiste dans les esprits, mais ni l'image ni l'adentite de la Mediterranée n'apparaissent en tant qu'entité globale. Pour faire partie de cente réalité de la géographie touristique, il faut que les pays du pourtour méditerranen forment ce bloc autour des valeurs qui les réunissent. L'organisation du tourisme euro-méditerranéenne, les actes, les actions bilaterales de partenariats economiques et environnementaux, etc, entre pays méditerranéens apportent déja quelques élé. ments de réponses au besoin d'unité et de fondement de cente entité méditerranéenne. e présent article est une réflexion sur la possibilité de mettre en place un projet commun entre pays méditerranéens à la recherche de cette unité. Nous abordons le sujet dans un premier temps en dressant un $*$ état des lieux $*$ du tourisme dans la région méditerranéenne (les tendances, les statistiques et les motivations louristiques, ainsi qu'une énumération sommaire des avantages et des inconvénients de la destination). A la lumière de ce qui précède, il faut faire ressortir les principaux besoins pour fixer des objectifs clairs et choisir les moyens d'action appropries pour atteindre ces objectifs. Finalement, nous dressons une liste des actions qui pourront être entreprises dans le cadre des rencontres méditerranéennes du tourisme.

\section{Avant-propos}

Saisir la réalité touristique de la région méditerranéenne passe par le rappel des principaux facteurs qui l'ont façonnée et qui ont rapproché - et rapprochent encore - les pays du Bassin méditerranéen en termes touristiques.

L'image de la région est fortement associée au soleil, à la tradition, à la culture et aux comportements méditerranéens. Tel est le « mythe de la Mediterranée \$. C'est d'abord une mer intếrieure entre l'Europe, l'Asie et l'Afrique ; elle communique avec l'Atlantique par le détroit de Gibraltar et avec la mer Rouge par le canal de Suez. C'est une mer chaude, à forte salinité et a faibles marées Sa profondeur maximale est de 4400 mètres. La péninsule italienne la divise en deux bassins : la Méditerranée occidentale, avec son annexe la mer Tyrrhếnienne, et la Méditerranée orientale qui se ramifie entre la mer Ionienne, la mer
Adriatique et la mer Égée. Elle fut le centre vital de l'Antiquité, jouant un rôle important dans l'évolution de l'histoire de l'humanité. Plusieurs civilisations ont eté créées et ont fleuri sur ses côtes: les maîtres de la Méditerranée étaient en même temps les maittres des voies de communication et du commerce entre $\mathrm{l}^{+}$Est et $\mathrm{I}^{+}$Ouest.

Cet héritage des grandes marques des civilisations égyptienne, phénicienne, grecque, romaine ou byzantine constitue un aspect culturel fondamental qui crée l'union des peuples méditerranéens, tandis que le climat, la gastronomie, l'art, le type d'habitat, le rythme de vie et des affaires sont là pour qualifier les atouts de chaque pays méditerranéen. Dès lors, la taille de la façade méditerranéenne n"apparaît pas comme l'aspect déterminant de l'appartenance à la Méditerranée. Il n'existe pas vraiment de définition d'un pays proprement méditerrancén car alors, il existerait aussi des normes d'appartenance à la Méditerranée.

La France, par exemple, est méditerranéenne car elle dispose, certes, d'une façade méditerranéenne, mais surtout d'un Sud méditerranéen réunissant tous les aspects méditerranéens et pesant beaucoup sur l'image de l'ensemble du pays. Le Portugal, bien que ne possédant pas de façade sur la Méditerranée, baigne dans la culture méditerranéenne par le passé de ses hommes. Malgré l'écart des flux touristiques et des devises entre les pays du Nord et du Sud, les régions méditerranéennes jouent un rồle considérable dans lă balance touristique de ces pays.

\section{L'état des lieux}

Comme le soulignait Francesco Frangialli' en 1993, \& la Méditerranée [...] est au- 
jourd'hui la plus importante destination touristique dans le monde, les pays qui la bordent reçoivent un tiers du total des arrivées de touristes, les zones proprement méditerranéennes de ces pays comptent pour un cinquième de ce même total $[. .$.$] \%.$ Cet écart s'explique par le fait que certains États, comme la France, ont une façade méditerranéenne, mais offrent d'autres produits touristiques (Paris, Disneyland, Châteaux de la Loire). Au total, les zones purement méditerranéennes accueillent 125 millions de touristes. Malgré cette première place et d'exceptionnelles réussites, cinq problèmes essentiels méritent d'être analysés, puis traités.

\section{Une croissance ralentie}

En valeur relative, toutes les études semblent montrer un déclin de l'aire méditerranéenne. Dans une étude de 1995 sur l'évolution des marchés touristiques, les experts de l'OMT (Organisation Mondiale du Tourisme) mentionnent que l'Asie de l'Est et le Pacifique restent la région du monde où la croissance touristique est la plus forte. Elle resterait supérieure à $10 \%$, contre moins de $5 \%$ pour l'Europe et le Moyen-Orient. L'Europe, qui en 1970 accueillait $62 \%$ du tourisme mondial, n'en a reçu que $49,5 \%$ en 1994 . Cette baisse relative n'est compensée que par une croissance des pays du Sud, où les flux touristiques présentent au contraire une grande stabilité,

Il convient cependant de s'assurer que cette perte des parts de marché ne sera pas un simple effet statistique. Une croissance forte en valeur absolue, portant sur des flux importants, peut être minorée en valeur relative par rapport à une croissance moindre, mais calculée sur des flux moins intenses. De 1000 a 1 100, la croissance est de $10 \%$, tandis que, de 50 à 100 , elle s'élève à $100 \%$.

\section{Des images ponctuelles,} mais pas d'image générale

La Méditerranée comprend des espaces touristiques qui ont une très bonne image de marque ponctuelle, comme Venise, la Costa Brava, la Tunisie phénicienne..., mais il n'y a pas d'image d'ensemble. La Méditerranée n'est pas en tant que tel un produit touristique, comme les Caraibes qui regroupent trente îles ou la zone $\mathrm{Pa}-$ cifique avec son organisation PATA (Pacific Asia Travel Association). La Méditerranée dispose, même si c'est assez timide, d'un organisme ${ }^{2}$ pour régler les problèmes juridiques et administratifs qui se posent au niveau des États. La formation et l'utilisation des nouvelles techniques de communication constituent les axes prioritaires des programmes en cours ou projetés.

\section{De rares innovations au plan des produits touristiques}

Les agents du tourisme méditerranéen ne semblent plus créer de nouveaux produits. Ils semblent vivre sur un acquis comprenant principalement le tourisme balnéaire et le tourisme culturel, généralement urbain. On ne dispose pas d'un outil de veille pour observer l'émergence de nouveaux produits, comme le phénomène des croisières ou le tourisme engendré par les festivités. Cette situation semble traduire un ralentissement de l'innovation en matière de tourisme, ce qui comporte des risques à moyen terme.

\section{Des sites surexploités et des aires vides}

Les types d'offre et de développement touristiques juxtaposent des aires périphériques dépourvues d'équipement et à l'écart des flux, ainsi que des espaces submergés qui doivent gérer le trop-plein. Il conviendrait d'étudier, puis de promouvoir des stratégies et des moyens de rééquilibrage.

\section{Le tourisme et l'environnement.}

Tourisme et environnement sont complémentaires et concurrents à la fois. Le tourisme méditerranéen est en partie guidé par la qualité de l'environnement, la beauté des sites, l'exploitation des ressources naturelles. Il s"agit sans doute plus d'un environnement perçu et vécu que de l'environnement réel. Quoi qu'il en soit de l'écart entre perception et réalité, l'environnement joue un rôle attractif certain. En mềme temps, le développement du tourisme modifie plus ou moins intensément l'environnement. Ces modifications sont encore mal connues. Cependant, toute une palette d'actions est envisageable entre les deux extrêmes que sont le laisser-faire et lat conservation. Les pollutions et l'urbanisation, étroitement liées, sont les deux plus grands problèmes.

Les foyers touristiques méditerranéens, mondialement connus, ne doivent donc pas masquer les difficultés actuelles. Certaines sont ponctuelles, mais d'autres concernent toute l'aire méditerranéenne. De plus, si de très nombreuses recherches menées séparément sont disponibles, les recherches-actions sur ce thème sont encore trop rares.

\section{Les objectifs et les priorités}

Les objectifs essentiels de notre démarche sont :

1- Dynamiser le tourisme dans la zone méditerranéenne.

2- Améliorer la fréquentation et les recettes touristiques dans les villes et les stations de la région.

3- Promouvoir le développement done l'emploi.

Pour les atteindre, nous avons établi cinq priorités :

\section{Créer une identitể touristique méditerranéenne}

Fernand Braudel, le grand historien de la Méditerranée, décrivait en 1985 la Méditerranée comme * une succession de mers [...] des civilisations entassées [...] de très vieilles choses encore vivantes qui côtoient l'ultramoderne $*$. Il ajoutait que : * [... $]$ dans son paysage physique comme dans son paysage humain, la Méditerranée carrefour, la Méditerranée hétéroclite se présente [...] comme une image cohérente, comme un système où tout se mélange et se recompose en une unité originale $[\ldots .$.$] .$ Il s'agit de faire émerger cette * image cohérente "et cette * unité originale * comme ont su le faire d'autres régions du monde aussi diversifiées que la nôtre.

Le cas des Caraíbes est à ce sujet exemplaire: formé de trente îles ou groupes d'îles (les Bermudes, la Jamainque, la Martinique, etc.), l'archipel apparaît comme la synthèse de cette pluralité insulaire. Les operateurs touristiques font 


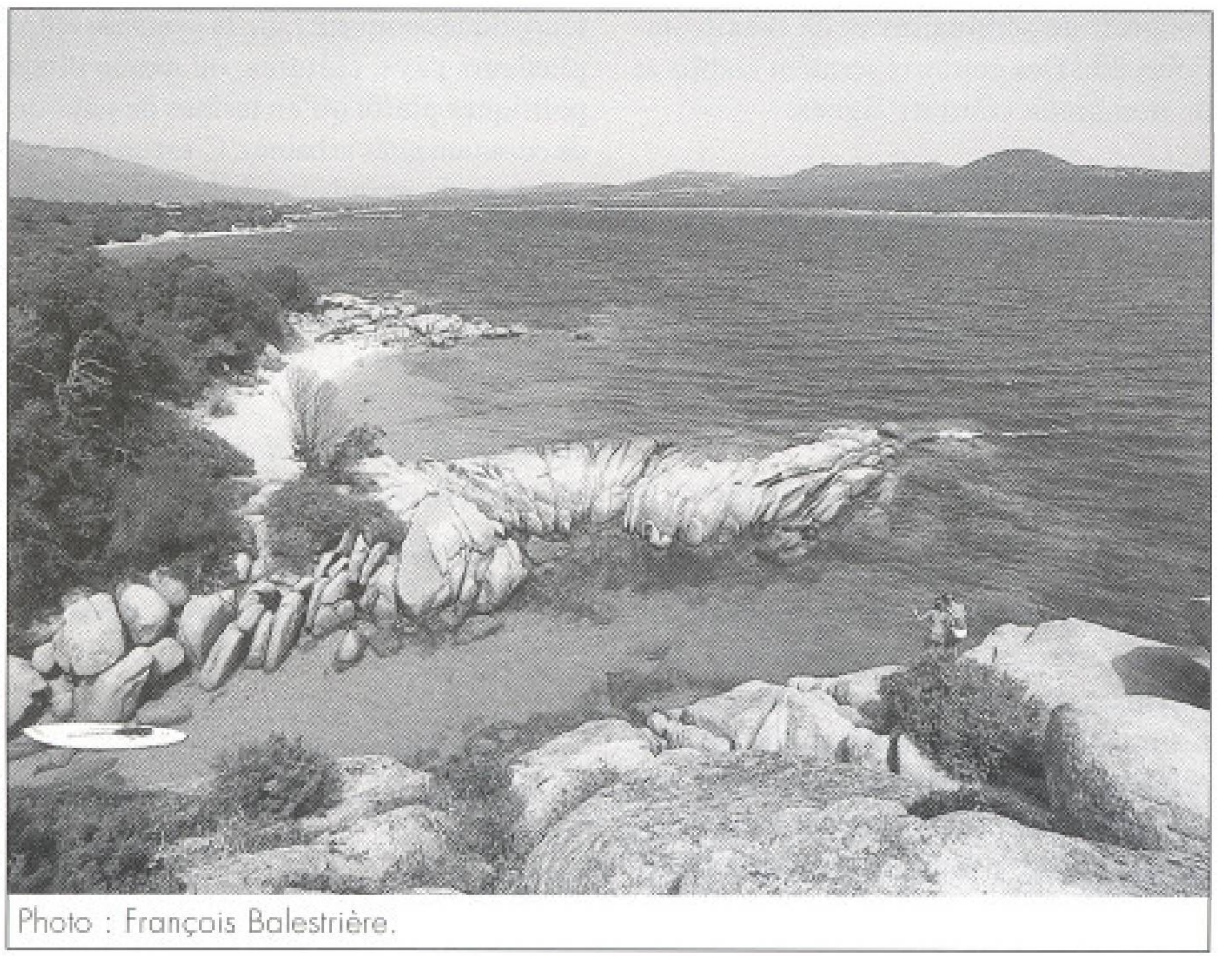

référence à l'ensemble et le programment comme une seule destination. La clientèle a ainsi l'impression qu'il s' agit d'un tout homogène.

\section{Rajeunir le produit et attirer les investisseurs}

Il conviendra de dresser l'inventaire des différents travaux à entreprendre et des améliorations à apporter au produit Méditerranée. Il faudra, par ailleurs, engager une vaste concertation entre les responsables de l'aménagement des pays concernés, les investisseurs privés et publics, les financiers et les intervenants susceptibles de gérer et de rentabiliser ces investissements. Il y aura de nombreux problèmes à régler sur le plan juridique : garantie des investissements, importation des capitaux et exportation des bénéfices, contrats des expatriés, etc.

\section{Conserver et protéger \\ l'environnement physique et culturel}

L'équilibre nécessaire entre qualité et quantité amènera sans doute à renforcer les procédures mises en place par le Plan Bleu pour réguler le développement des constructions le long des $46000 \mathrm{~km}$ de côtes du Bassin méditerranéen. De plus, un soin particulier sera apporté à la préservation des monuments, dont la visite a toujours été une des principales motivations touristiques, mais aussi à la préservalion des cultures, surtout des cultures minoritaires : petits groupes ethniques en voie de disparition, langues peu ou plus parlées, savoir-faire artisanaux, gastronomiques et agricoles, religion et rites, etc. Comme l'affirmait Braudel, il faut à tout prix préserver et conserver cette diversité qui se fond en une unité ; elle est notre richesse.

\section{Organiser la formation des gens et les transferts de technologies}

Le décalage entré les pays méditerranéens d'Europe et ceux d'Afrique et du ProcheOrient est évident et il apparaît dans tous les domaines : fréquentation (66 millions d'arrivées internationales en France, 3,5 millions en Tunisie), équipements, capacité à gérer, recettes, communication, infrastructures, etc. La cohérence et l'unité de la destination ne pourront être atteintes que si ce décalage est réduit. Il faudra encourager les échanges de main-d'œeuvre, la mise en place de stages, de formations initiales, continues et professionnelles à tous les niveaux, tout comme les transferts de technologies qui seront pris en charge par les États, les collectivités publiques et les groupes industriels.
Favoriser la coopération entre les intervenants touristiques publics et privés de tous les pays méditerranéens

Il apparaît primordial de donner à tous les intervenants la possibilité légale et la volonté de travailler et de promouvoir ensemble leur région, leur industrie et leur innovation. L'identité touristique méditerranéenne sera le résultat d'un certain \& vouloir vivre et travailler ensemble *.

Elle émergera lorsque, par exemple, des compagnies aériennes nationales signeront un accord pour la création d'un \& Mediterranean Air Pass s qui permette à un touriste d'acheter des coupons de voyage valides auprès de plusieurs compagnies desservant les différentes destinations de la région, lorsque des voyagistes produiront une brochure \& Méditerranée \&, ou encore quand les chaînes hôtelières proposeront des prix similaires et des bons de séjours acceptés par les unités hôtelières des diverses chaînes de plusieurs pays. Parallèlement, les administrations de chaque pays devront s'efforcer de régler de nombreux autres problèmes (les visas, les douanes, etc.) et les gouvernements de faciliter les relations culturelles et politiques. Toutes ces questions, bien qu'elles affectent l'organisation du tourisme, le dépassent de très loin ; $\mathrm{c}^{+} \mathrm{est}$ pourquoi nous les laisserons aux spécialistes et nous en tiendrons aux objectifs ci-dessus mentionnés qui nous semblent déjàt très ambitieux.

\section{Les moyens}

Sans nous substituer à l' action de l'Orgatnisation du Tourisme Euro-Méditerranéen (OTEM) qui est efficace aux plans administratif et inter-étatique, si nous désirons atteindre les objectifs fixés, il convient d'imaginer un espace de rencontres d'une toute autre ambition, innovant tant dans ses méthodes que ses stratégies. Un espace où tous les acteurs du tourisme méditerranéen quelles que soient leurs spécificités et leur situation dans la chaîne économique, auront la possibilité d'élaborer ensemble leurs projets et leurs politiques de développement. Cette initiative irait tout à fait dans le sens des décisions de la commission de l'Union Européenne qui incite â élargir le champ de coopération 
entre les pays de l'Union et les pays tiers méditerranéens ${ }^{3}$, notamment à l'intérieur d'actions de coopération interrégionale décentralisée.

Plus encore, elle apparaît comme particulièrement conforme aux conclusions du Conseil européen de Cannes (1995) : « Le Conseil européen réaffirme l'importance stratégique qu'il attache à ce que les relations de l'Union Européenne avec ses partenaires de la Méditerranée prennent une nouvelle dimension permettant, notamment, la construction de zones de prospérité partagée $\%$. La création de cet espace de rencontres que nous préconisons, en répondant aux priorités fixées par la Commission qui portent sur * les opportunités de partenariat » et sur \& la promotion du commerce et de l'exportation $\%$, répond tout autant aux nécessités de développement de l'industrie touristique du Bassin méditerranéen.

Nous prévoyons, en effet, la mise en place d'une plate-forme de concertation entre les partenaires du tourisme méditerranéen : institutionnels, personnalités politiques, collectivités territoriales, investisseurs. financiers, intervenants divers.

\section{Les rencontres méditerranéennes du tourisme}

Ces rendez-vous du tourisme méditerranéen se tiendraient à intervalles réguliers (chaque année sans doute) :

- soit en un lieu fixe, ce qui faciliterait évidemment la logistique :

- soit alternativement dans des villes des rives nord et sud du Bassin méditerranéen, ce qui contribuerait à améliorer le processus de coopération.

Ces rencontres s'échelonneraient sur une semaine et on y aménagerait quatre types d'actions opérationnelles :

\section{Une bourse du tourisme}

Pays d'accueil, stations de tourisme, hôteliers, restaurateurs, transporteurs, agents réceptifs, etc., rencontreraient acheteurs, tour opérateurs, autocaristes, clubs, associations de pays émetteurs, agents de voyages, entreprises, organisateurs de congrès, de séminaires et de manifestations, etc. Des contacts seraient établis et de nombreux contrats signés.

\section{Un forum des investissements}

Collectivités locales, institutionnels, investisseurs et financiers jetteraient les bases de programmes d'investissement et de développement.

\section{Un salon de la formation}

Les principaux établissements publics et privés d'enseignement du tourisme y exposeraient. Ils pourraient ainsi établir pour l'échange de stagiaires entre opérateurs des pays concernés et établissements d'enseignement. Experts, consultants, organismes de formation continue, etc., proposeraient aux entreprises et aux institutions des séminaires, des cycles, des formations de durée variable. Bref, un lieu de rencontres pour l'emploi serait aménagé.

\section{Des cycles de conférences}

Ils permettraient la mise à niveau des partenaires sur les sujets les plus divers : nouvelles technologies, commercialisation, ressources humaines, géopolitique, sciences économiques, etc. Des experts rendraient compte de leurs expériences, de leurs réussites... et de leurs échecs.

\section{Conclusion}

C'est vraisemblablement lors de ces rencontres qu' un véritable esprit de partenariat pourrait naître. Les pays du monde méditerranéen, dont certains vivent presque exclusivement du tourisme, se font concurrence les uns les autres et font plus d'efforts pour tenter de détourner la clientèle de leurs concurrents proches que pour capter de nouveaux marchés. En unissant leurs efforts, ces pays obtiendraient certainement de biens meilleurs résultats. C'est en facilitant les contacts, en permettant aux gens de tourisme de travailler ensemble, en les incitant à promouvoir la région et non plus seulement leur station ou leur pays, que nous retrouverons l'unité originale de Braudel.

L'expérience prouve que le phénomène de mondialisation du tourisme amène à penser l'évolution et le développement de l'industrie touristique en termes de zones touristiques pouvant faire la synthèse entre plusieurs pays, cultures, ou même blocs politiques plutôt qu'en termes de pays ou de communautés urbaines. $\mathrm{C}^{*}$ est en mettant en place de grands rendez-vous internationaux du type des rencontres méditerranéennes du tourisme que nous préconisons que nous faciliterons le progrès et le développement du tourisme.

Samir Khlifi est docteur en géographie du tourisme et enseignant-chercheur à l'Université de Nice - Sophia Antipolis.

\section{Notes}

1 Secrétaire général de l'Organisation Mondiale du Tourisme (OMT).

2 L'année 1996 correspond à la mise sur pied de l'Organisation du Tourisme Euro-Méditerranéen (OTEM), association internationale de Droit Marocain dont le siège est à Rabat, la direction opérationnelle et le siège de la région Occidentale à Nice, et le siège de la région Orientale à Damas.

3 Coopération interrégionale décentralisée en Méditerranée (juillet 1995), Note d'information, Programme OUVERTUREMED, contexte, objectifs et organisation.

\section{Bibliographie}

Braudel, Fernand (1985), La Méditerranée, l'espace et l'histoire, Flammarion, Paris.

Charte du Bassin méditerranéen (1994), Forum international, Étude sur la faisabilité sur l'implantation à Nice d'une agence méditerranéenne du tourisme.

Conseil Européen de Cannes (1995), Conclusions de la présidence, 26-27 juin.

Frangialli, F. (1994), * Le tourisme en Méditerranée : l'enjeu du développement durable pour une destination majeure fragile $*$, Espace, $n^{\circ} 126$.

Gazette officielle du tourisme (1998).

Organisation Mondiale du Tourisme (OMT) (1995), Étude sur l'évolution des marchés touristiques. 